\title{
A Strategy for Selective Low Vacuum Imaging and Focused Ion Beam Milling of Multi-Layer Optoelectronic Polymer Devices
}

\author{
D.J. Stokes, ${ }^{*}$ C.M. Ramsdale, ${ }^{* *}$ H. Mulders ${ }^{* * *} \&$ A.M.Donald ${ }^{*}$ \\ * Biological \& Soft systems, Cavendish Laboratory, Madingley Road, Cambridge, CB3 0HE, UK \\ ** Plastic Logic Ltd, Cambridge Science Park, Milton Road, Cambridge, CB4 OFX, UK \\ **** FEI Company, Building AAE, Achtseweg Noord, PO Box 80066, Eindhoven, The Netherlands
}

Polymer semiconductor devices are being developed as low-cost, flexible alternatives to traditional silicon-based devices in areas such as display technology. Imaging of certain polymer components has previously been demonstrated using low vacuum imaging [1, 2], and we extend this methodology to more complex, patterned multi-layer structures, where nanoscale pattern alignment and homogeneity of interfaces are critical characterisation parameters.

A typical device may consist of several thin layers: a metallic source/drain, semi-conducting polymer, dielectric material (e.g. insulating polymer) and a metallic gate. The polymer and dielectric are laid down by spin coating/inkjet printing onto an electrically insulating substrate. We placed such a device in an FEI Quanta 3D DualBeam ESEM. In addition to the flexibility of low vacuum imaging, we can perform site-specific cross-sectioning with a focused ion beam (FIB). A method for charge stabilising specimens during FIB milling in high vacuum is described elsewhere [3].

Figures 1 and 2 demonstrate the selective use of beam energy to help identify areas of interest. At 30 $\mathrm{keV}$ (fig. 1) an underlying layer of interdigitated $\mathrm{Au}$ is visible, while at $15 \mathrm{keV}$ (fig. 2) the signal from the upper gate dominates, completely masking the layers underneath. Thus we can take advantage of the low electron stopping powers of organic matter and the production of backscatterderived SE signals from Au to selectively form these images, and low vacuum mode for chargecontrolled imaging irrespective of beam energy.

Figure 3 shows just the interdigitated region. The layers on top of this structure, not apparently visible in the image, have a total thickness of $900 \mathrm{~nm}$. The interdigitated region cannot be observed at low beam energies and, similarly, cannot be seen using an ion beam-induced signal due to small primary beam penetration depths. Precise selection of the milling site was therefore reliant on reference to the high-energy electron-induced image (an advantage of dual beam over single beam FIB), as was the alignment of both beams at the point of interest.

Figure 4 is a low vacuum image of a FIB-milled cross-section through the device, at $52^{\circ}$ tilt. In particular, it shows part of a now-exposed Au source or drain line from the interdigitated region, thickness $\sim 50 \mathrm{~nm}$. In this case, a more surface-sensitive beam energy ( $5 \mathrm{keV}$ ) was used for imaging.

\section{References}

[1] Ramsdale, C.M. et al Physica E, 14 (1-2), (2002) 268-271

[2] S.W. Williams \& A.M. Donald, J. Microsc 216, (2004) $241-8$

[3] D.J. Stokes, F. Morrissey, W.R. Knowles \& A.M. Donald, elsewhere in these proceedings

[4] The financial support of FEI Company is gratefully acknowledged 

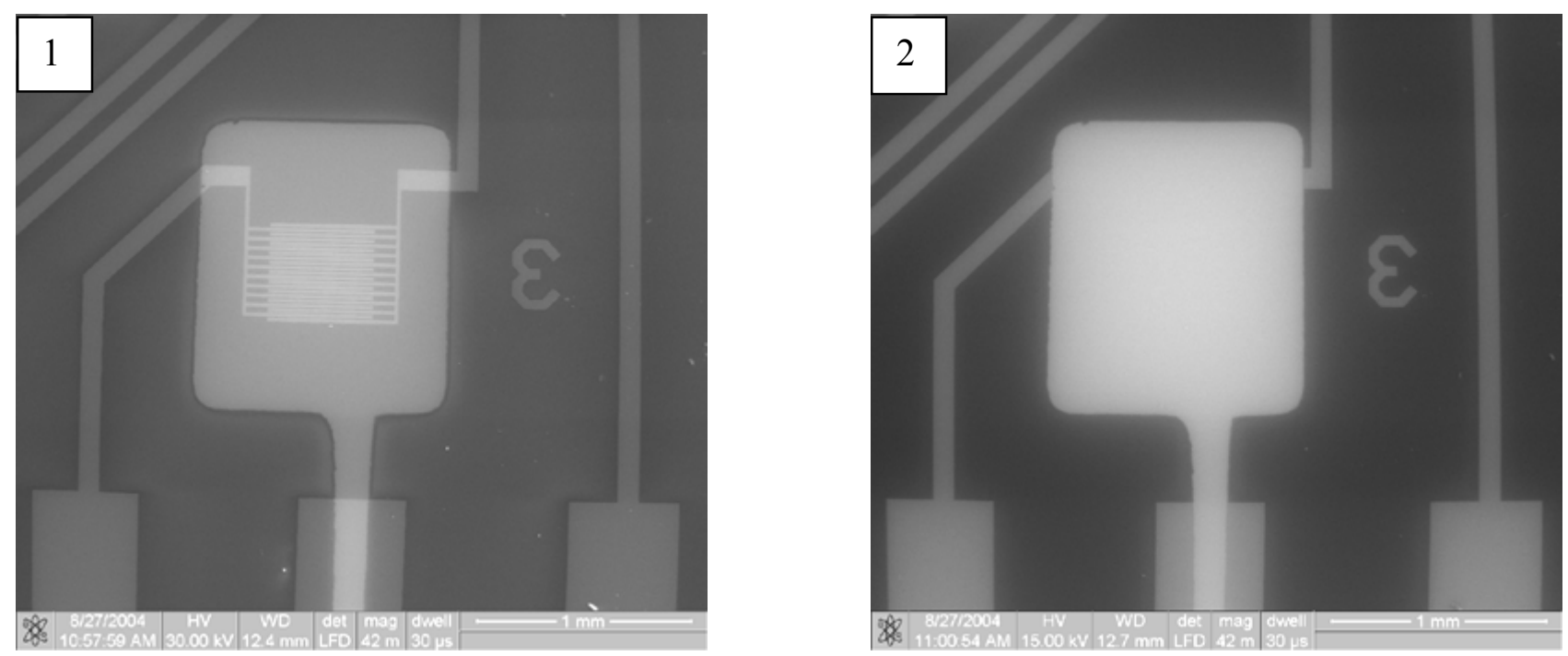

Fig. $1.30 \mathrm{keV}$ low vacuum image. Interdigitated sources and drains are visible beneath the multilayered structure (polymer, dielectric and gold, total thickness $900 \mathrm{~nm}$ ).

Fig. 2. As fig. 1, but with beam energy $=15 \mathrm{keV}$. Sub-surface detail is still visible through the multilayers at this energy, although the signal from the interdigitated region is no longer seen.
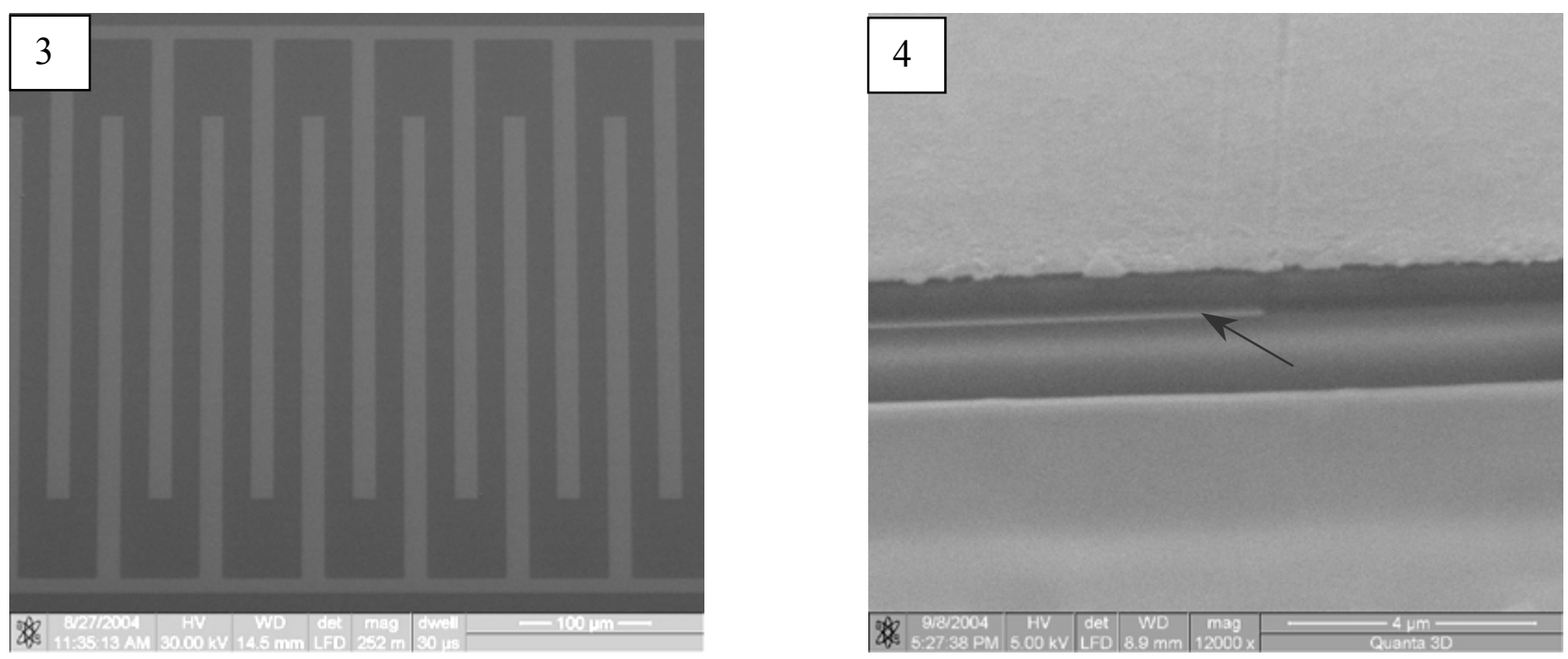

Fig. 3. Higher magnification image ( $30 \mathrm{keV}$, low vacuum) of the interdigitated sources and drains. Note that the structure is rotated $90^{\circ}$ relative to fig. 1 .

Fig. 4. FIB cross-section through the device. High magnification image at $52^{\circ}$ tilt, $5 \mathrm{keV}$. Part of an exposed $50 \mathrm{~nm}$-thick source/drain line is seen edge-on (arrowed). The adjoining semi-conducting polymer is $\sim 50 \mathrm{~nm}$ thick, the dielectric layer above is $\sim 800 \mathrm{~nm}$ thick and the overlying gold pad is $\sim 50 \mathrm{~nm}$ thick. 